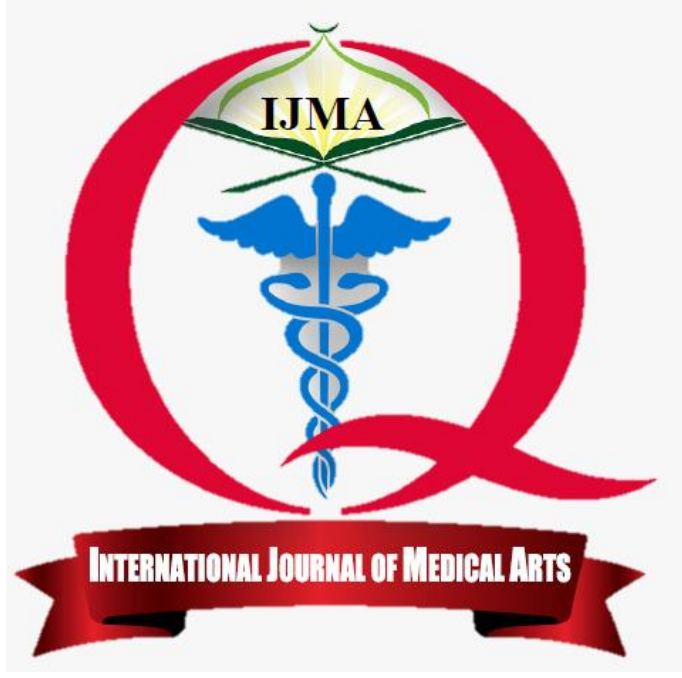

Available online at Journal Website

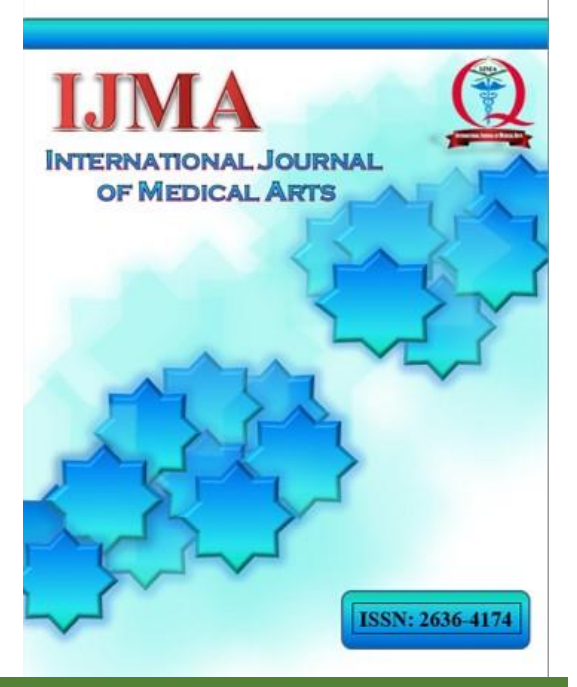

Original article

\title{
Feasibility and Accuracy of Two-and Three-Dimensional Transthoracic Echocardiography in patients with Right Atrial Dilatation using the Right Parasternal Approach
}

\author{
Khaled Ibrahim Najeeb; Mohamed Sayed Bashandy; Reda El-Sayed Abd Elfattah
}

Cardiology Department, Faculty of Medicine, Al-Azhar University (Damietta), Egypt.

\section{Corresponding author \\ Reda El-Sayed Abd Elfattah \\ Residnet at Cardiology Department; \\ Al-Azhar Faculty of Medicine (Damietta), Egypt. \\ Email: www.reda2020@gmail.com}

Received at: May, 15, 2019

Revised at: June 25, 2019

Accepted at: June 26, 2019

Available online at: July 7, 2019

Published: July 7, 2019
Background: Echocardiography increasingly used for diagnosis of right atrial dilatation. Three dimensional is superior than two dimensional echocardiography. However, advances and new windows continues to emerge to increase its accuracy

Aim of the work: to show the feasibility and accuracy of right parasternal approach and its usefulness in qualitative and quantitative assessments of right atrium in patients with right atrial dilatation in comparison with transesophageal approach.

Patients and Methods: The study included thirty patients with right atrial dilatation. All patients underwent full history taking, clinical, 12-lead electro-cardiography and echocardiography. Transesophageal Echocardio-graphy was used as a gold standard for the accuracy of the right parasternal approach.

Results: The study includes 30 patients with right atrial dilatation the right parasternal (RPS) view obtained in 18 patients $(60 \%)$, and not obtained in 12 patients (40\%). There was significant decrease of SVC anteroposterior minimal diameter (Min AP), crista terminalis (CT) width, CT length, right atrial appendage (RAA) base Maximum diameter and RAA base Minimum diameter, in 2D Echocardiography when compared to $3 \mathrm{D}$ Echocardiography. On the other side, there was no statistically significant difference in Right parasternal approach (3D) and Transesophageal approach.

Conclusions: Parasternal approach is feasible in imaging right heart structures. In addition, three dimensional TTE had an incremental value over two dimensional TTE in the assessment of these structures

Keywords: Echocardiography, Parasternal Approach; three-dimensional; two dimensional; right atrial dilatation.

\section{DOI: $10.21608 /$ /ijma.2019.39236}

This is an open access article under the Creative Commons license (CC BY) (https://creativecommons.org/licenses/by/2.0/)

Please cite this article as: Najeeb KI, Bashandy MS, Abd Elfattah RE. Feasibility and Accuracy of Two-and Three-Dimensional Transthoracic Echocardiography in patients with Right Atrial Dilatation using the Right Parasternal Approach. IJMA 2019; 1(1): 58-64. 


\section{Introduction}

Size of the right atrium is clinically important as it reflects the function of the right ventricle with clinical outcomes in many conditions such as tricuspid regurgitation, pulmonary hypertension, and right heart failure ${ }^{[1]}$.

The noninvasive assessment of right atrial structures as superior vena cava (SVC), crista terminalis (CT), and the right atrial appendage (RAA) has clinical implications in determining the pressure of the right heart in adults with heart failure, pulmonary hypertension, and pericardial effusion in whom the imaging of inferior vena cava is difficult due to poor subcostal window as in obesity or previous surgery of the abdomen. Planning electrophysiological mapping and ablation techniques is crucial to detect thrombi in the right atrium or right atrial appendage for patients complaining of atrial fibrillation. However, these structures are difficult in imaging by using standard two-dimensional transthoracic echocardiography (2DTTE) ${ }^{[2]}$.

Parasternal approach is another approach, in which the imaging of these structures is possible. However, this approach is not described frequently in the echocardiographic literature or current imaging guidelines ${ }^{[3]}$.

Right parasternal examination is carried out in right lateral decubitus position to permit for lateral displacement of the right lung and to open up a window on the right side of the sternum. The transducer is usually positioned on the right parasternal intercostal spaces next to the sternal border to allow imaging of superior vena cava, right atrial appendage and other nearby structures. In patients with enlarged right heart or aorta or pericardial effusion, the view could be successfully attained more often and this can be explained by the tendency of such patients to displace right lung laterally and opening up this window. Also it was found that a number of patients without these anomalies can also be investigated using this transthoracic technique ${ }^{[4]}$.

Although transthoracic two-dimensional echocardiography (TT-2DE) is the most common noninvasive diagnostic modality, opinions of experts are required for data explanation because of the intra-cardiac complex anatomy. This is considered a limitation when using two dimensional transthoracic echocardiography alone and often leads to varying explanation by different observers. The previously mentioned limitation of
TT-2DE alone are overcome by the usage of real time transthoracic three-dimensional echocardiography (RTT-3DE), which offers meticulous assessment of right atrial structures in right parasternal approach ${ }^{[5]}$.

The structures of right heart could be identified by the usage of three-dimensional transesophageal echocardiography (3DTEE). It is a semi-invasive technique with well-known drawbacks, as right atrial appendage could not be visualized all the time, event it could be imaged by 2DTEE. Also, a small number of patients undergoing TEE in clinical practice, so, a need is crucial to try imaging these structures by TTE ${ }^{[6]}$.

\section{Aim of the work}

The aim of this study was to show the feasibility and accuracy of right parasternal approach and its usefulness in qualitative and quantitative assessments of right atrium in patients with right atrial dilatation in comparison with transesophageal approach (The Gold standard).

\section{Patients and Methods}

This is a prospective observational study which included thirty patients with right atrial dilatation presented to the Cardiology Department of Al-Azhar University Hospital in New Damietta; the study was carried out over a period of eight months from August 2018 to July 2019.

Population with right atrial dilatation assessed by standard $2 \mathrm{D}$ echocardiography were included in the study. The size of the right atrium was determined by measurement of its long-axis dimension in apical four-chamber view. This parameter is easily obtained and delivers a good estimation for right atrial size. The upper limit of right atrial long axis is $45 \mathrm{~mm}^{[7]}$.

The reference values of right atrium length were described by Kawel-Boehm et al. ${ }^{[8]}$. Accordingly, normal length was ranged from 29 to $45 \mathrm{~mm}$; mild dilatation (46-49 $\mathrm{mm}$ ); moderate dilatation $(50-54 \mathrm{~mm})$ and severe dilatation $(\geq 55$ $\mathrm{mm})$

In our study we excluded the patients with poor conventional echo window, coagulopathy, prior esophageal surgery or known esophageal stricture and esophageal varices or tumor.

The study protocol was approved by the Local Research and Ethics Committee of Al-Azhar University, Faculty of Medicine (Damietta). 
All patients signed an informed consent to participate in the study after full explanation of the study procedures. Then, all were submitted to full history taking (patient age, gender, symptoms suggesting- cardiac dysfunction [chest pain, dyspnea, orthopnea, paroxysmal nocturnal dyspnea]), routine clinical examination which included general examination [signs of cardiac dysfunction] and systematic cardiovascular examination. Then, resting 12-Leads (ECG) was performed for detection of RA enlargement (RAE). Finally, all were submitted to transthoracic Echocardiography which carried out on two stages: the first is resting conventional Trans-Thoracic Echocardiography done at echocardiography Lab of Cardiology Department at Al-Azhar University Hospital (New Damietta) using Philips IE 33 . The probe used was the $X$ 5-1 phased array sector probe (frequency range 1.5-4.3 MHz). Images were acquired with patients in the left lateral position according to the guideline of the American society of Echocardiography ${ }^{[9]}$. The second stage is the right parasternal approach as described by Arisha et al. ${ }^{[2]}$

Briefly, the patient assumed a right-lateral decubitus position that may vary from supine to more than 90 degrees. An angle of approximately 45 degrees was the most commonly used. The transducer index marker was directed either toward the patient's head or to the patient's left. This transducer orientation allows the anatomy that is nearest to the marker to be displayed on the right of the echocardiographic video monitor. The actual window may range from the xiphoid process to the subclavicular region. The right axilla is the lateral limit. Generally, the sequence of RPS views that we try to obtain started with a long-axis view of the ascending aorta. Next, the aortic arch view, followed by the long axis view of the atrial septum as well as the superior, inferior vena cava, CT and RAA. After these RPS views from the long-axis plane, the transducer is rotated approximately 90 degrees to obtain the short-axis views. The RPS short-axis examination generally starts with a short-axis view at the base of the heart. First, the atrial septum and aortic valve views are attempted followed by the short-axis view of the superior vena cava (SVC), CT and RAA. The short-axis view of the left and right ventricles completes the examination.

Transesophageal Echocardiography was used as a gold standard for the accuracy of the right parasternal approach in patients with right atrial dilatation. All subjects were examined in the conscious and fasting state using $10 \%$ lidocaine spray and lidocaine gel to anesthetize the posterior pharyngeal wall. Sedation when needed was achieved by (midazolam Hydrochloride). The technique was carried out with constant ECG and saturation monitoring using a multi-plane TEE probe. The TEE probe (X7-2) was introduced. The TEE examination was done emphasizing on the next measurements: The maximum and minimum antero-posterior and lateral lumen diameters of the SVC were measured. Also, the full length and width of crista terminals, the maximum and minimum inner length, and width of RAA base were measured in mid oesaphageal (bicaval) 90 right view.

Statistical analysis: A "Data Collection Form" was prepared and used to collect different demographic, clinical and radiological data. Then, data transferred to Statistical Package for Social Sciences (SPSS version 19.0) program to be analyzed. Different descriptive statistics were used (e.g., mean, standard deviation, minimum and maximum for quantitative parameters; number and percentages for qualitative parameters). Comparison between quantitative data was done using non-paired student $(\mathrm{t})$ test and $\mathrm{P}$ value was considered significant if $<0.05$.

\section{Results}

In the present study, $76.7 \%$ of patients were males; the mean age of studied populations was $39.07 \pm 5.79$ (range 30- 52). In addition, $46.7 \%$ of patients had moderate right atrial dilatation, $30 \%$ had severe dilatation, mean size of it was \pm 4.03 with range from 46 to 57 (Table 1).

The study includes 30 patients with right atrial dilatation the RPS view obtained in 18 patients $(60 \%)$, and not obtained in 12 patients $(40 \%)$ (Data not tabulated).

In the present work, the structures obtained by 2D and 3D- echocardiography using the RPS approach were presented in table (2).

In the present work, there was significant decrease of SVC minimal diameter (AP) in $2 \mathrm{D}$ when compared to 3D echocardiography. In addition, there was significant decrease of CT width, CT length, RAA base maximum diameter and RAA base minimal diameter, in $2 \mathrm{D}$ when compared to 3D (Table 3). On the other side, there was no statistically significant difference in Right parasternal approach (3D) and Transesophageal approach (Gold standard) (Table 4). 

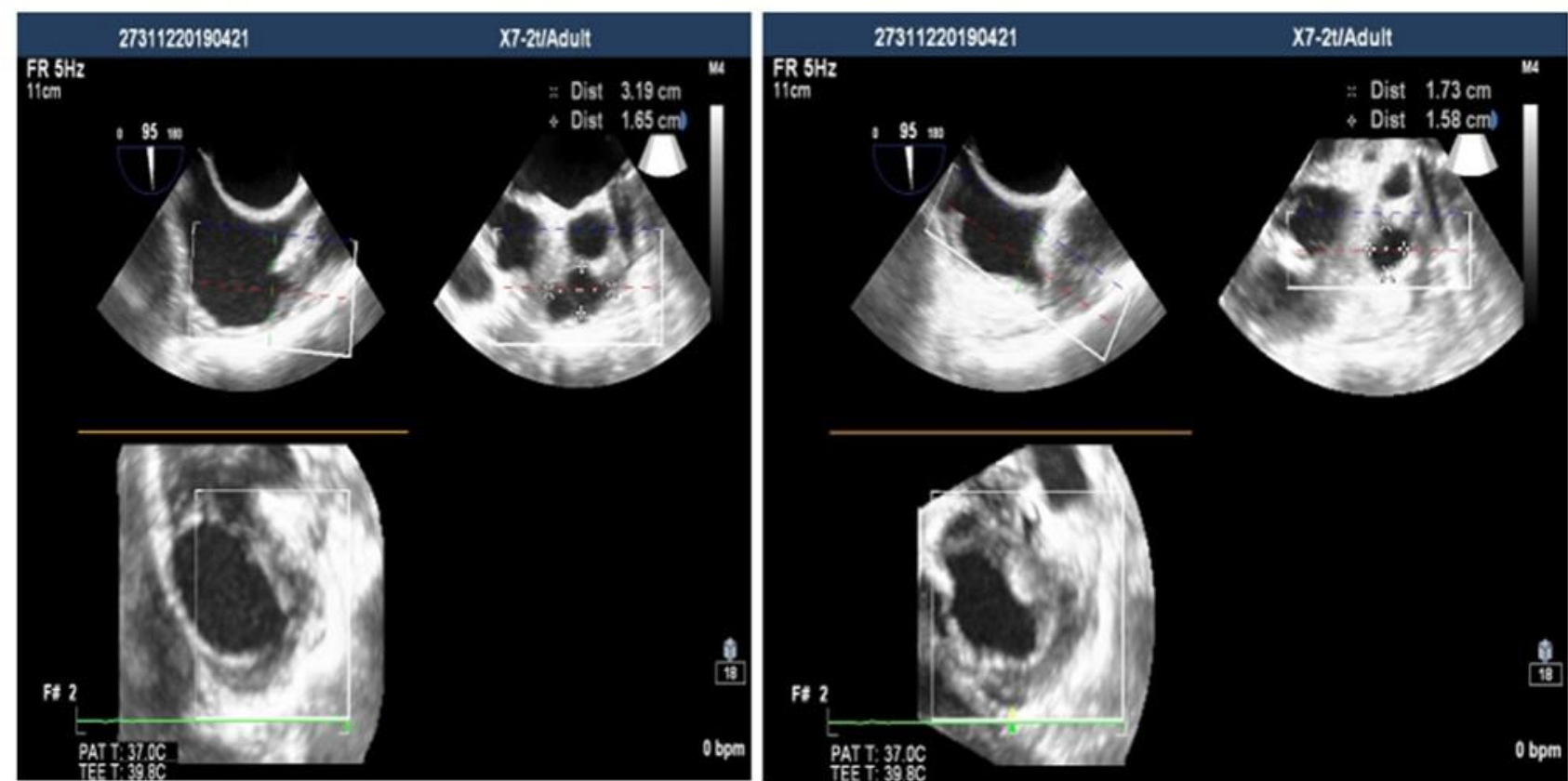

Figure (1) TEE mid esophageal bicaval view showing RAA base length and width maximum and minimum diameters.
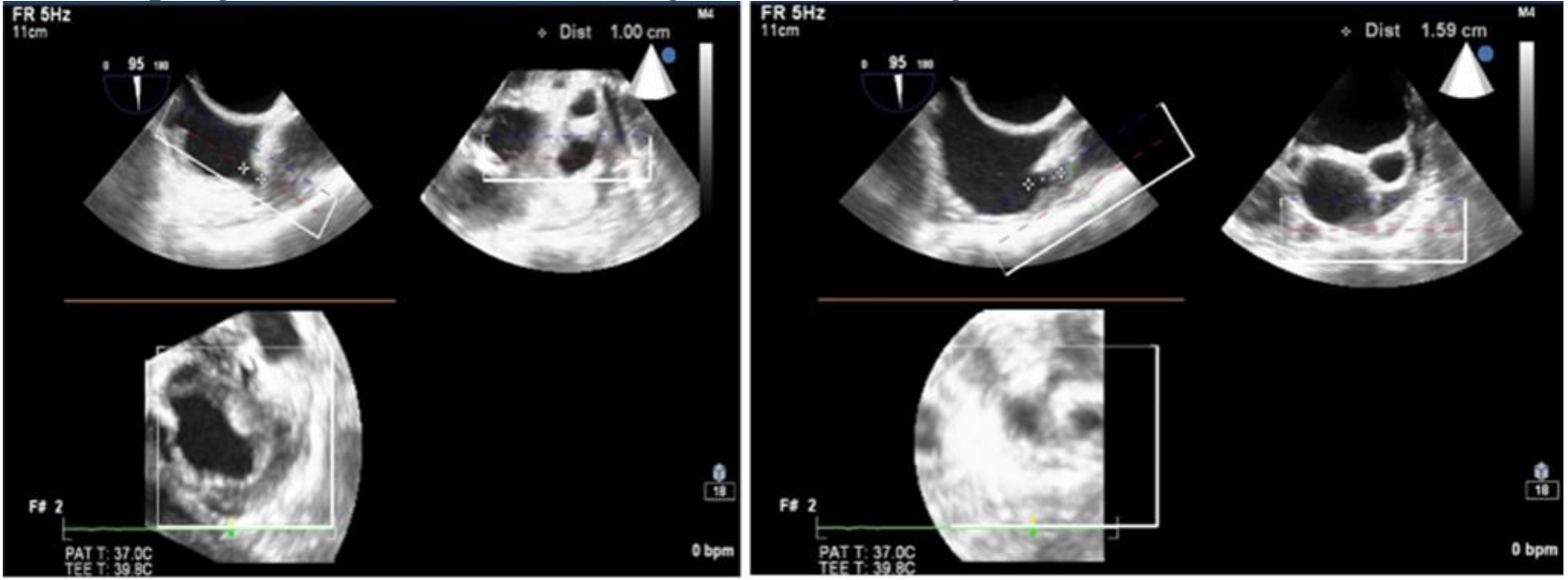

Figure (2): TEE mid esophageal bicaval view showing RAA depth maximum and minimum diameters.
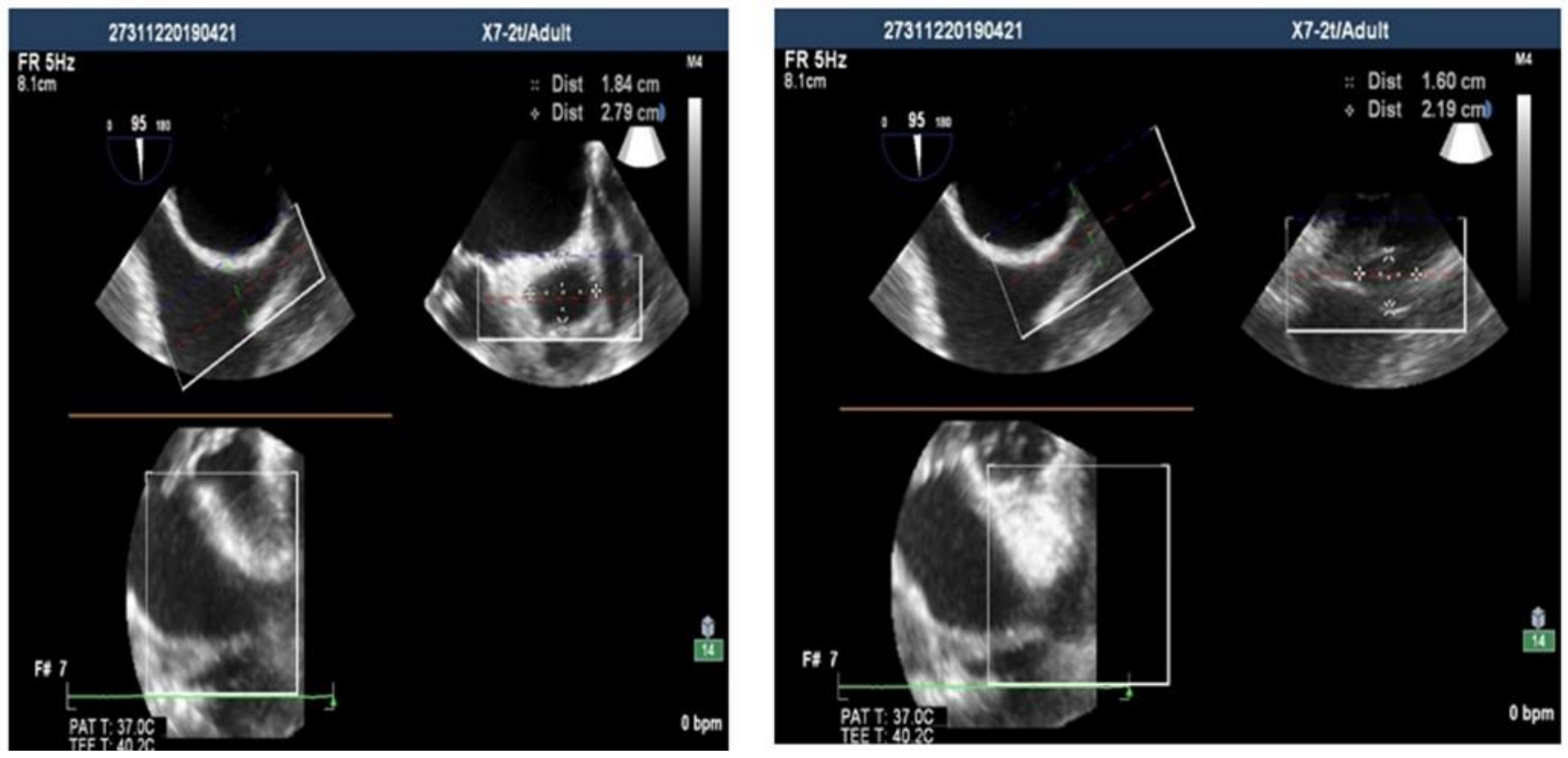

Figure (3): TEE mid esophageal bicaval view showing SVC maximum and minimum diameters.

Table (1): Demographic data and right atrial size (long axis dimension) among studied populations

\begin{tabular}{|c|c|c|}
\hline \multicolumn{2}{|c|}{ Statistics } \\
\hline \multirow{2}{*}{ Sex } & Female & $7(23.3 \%)$ \\
\cline { 2 - 3 } & Male & $23(76.7 \%)$ \\
\hline Age & Mean \pm SD & $39.07 \pm 5.79$ \\
\cline { 2 - 3 } & Range & $30-52$ \\
\hline Right atrial size \\
(Long axis dimension) & Mild & $74(46.7 \%)$ \\
\cline { 2 - 3 } & Moderate & $9(23.3 \%)$ \\
\cline { 2 - 3 } & Severe & $51.00 \pm 4.03$ \\
\cline { 2 - 3 } & Mean \pm SD & $46-57$ \\
\cline { 2 - 3 }
\end{tabular}


Table (2): 2D and 3D echocardiography and TEE among studied populations

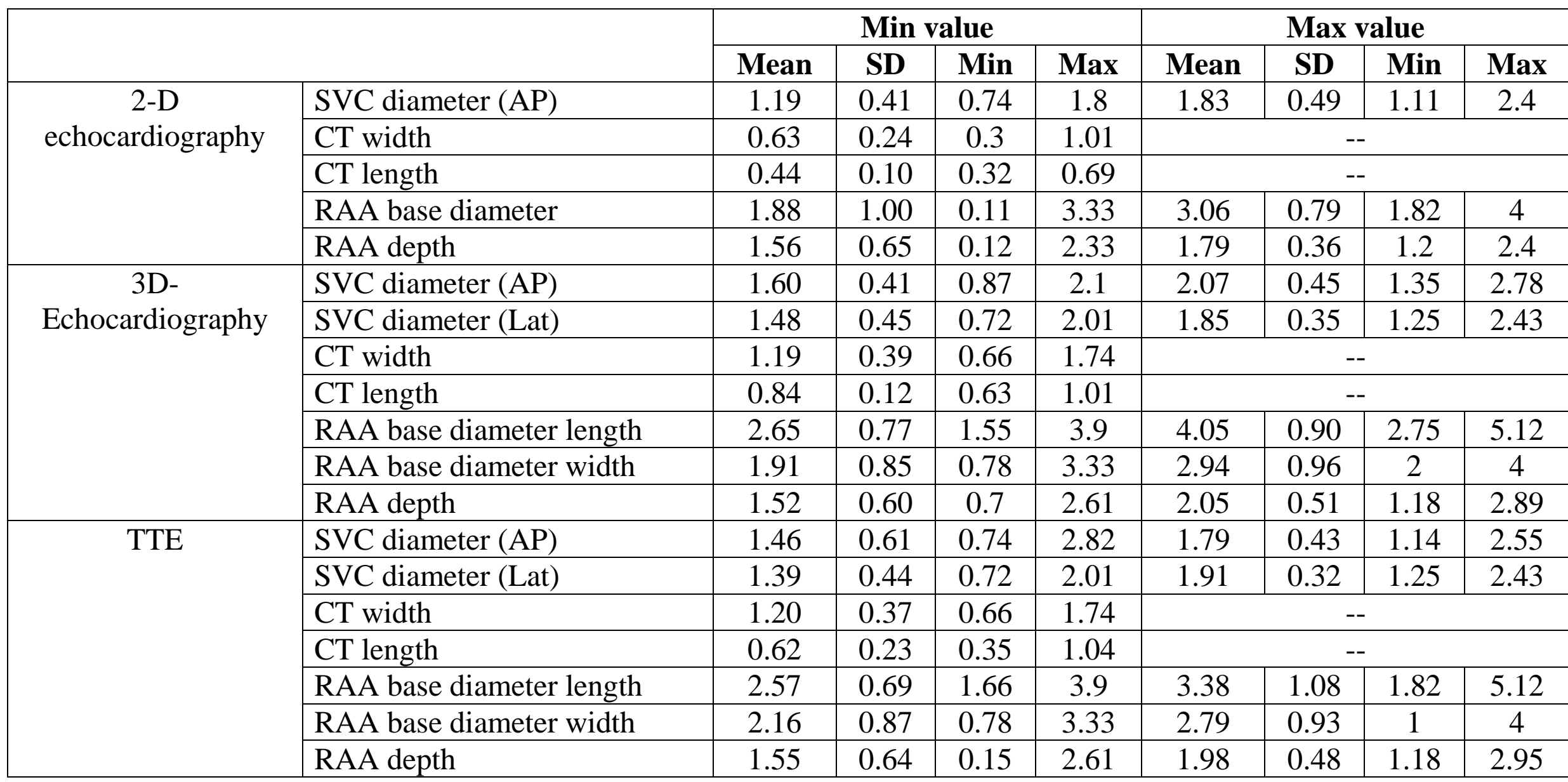

Table (3): Comparison between $2 \mathrm{D}$ and $3 \mathrm{D}$ echocardiography among studied populations

\begin{tabular}{|l|c|c|c|c|c|c|}
\hline \multirow{2}{*}{} & \multicolumn{2}{|c|}{ 2D } & \multicolumn{2}{c|}{ 3D } & \multicolumn{2}{c|}{ Independent t test } \\
\cline { 2 - 7 } & Mean & SD & Mean & SD & t & P value \\
\hline SVC diameter Max(AP) & 1.83 & 0.49 & 1.98 & 0.65 & -0.796 & 0.431 \\
\hline SVC diameter Min(AP) & 1.19 & 0.41 & 1.64 & 0.50 & -2.932 & $\mathbf{0 . 0 0 6} *$ \\
\hline SVC diameter Max (Lat) & -- & -- & 1.87 & 0.32 & -- & -- \\
\hline SVC diameter Min (Lat) & -- & -- & 1.56 & 0.52 & -- & -- \\
\hline CT width & 0.63 & 0.24 & 1.19 & 0.39 & -5.246 & $\mathbf{0 . 0 0 1 *}$ \\
\hline CT length & 0.44 & 0.10 & 0.84 & 0.12 & -11.068 & $\mathbf{0 . 0 0 1 *}$ \\
\hline RAA base diameter Max & 3.06 & 0.79 & 4.05 & 0.90 & -3.508 & $\mathbf{0 . 0 0 1 *}$ \\
\hline RAA base diameter Min & 1.88 & 1.00 & 2.65 & 0.77 & -2.598 & $\mathbf{0 . 0 1 4} *$ \\
\hline RAA depth Max & 1.88 & 0.36 & 2.05 & 0.51 & -1.181 & 0.246 \\
\hline RAA depth Min & 1.56 & 0.65 & 1.52 & 0.60 & 0.176 & 0.861 \\
\hline RAA base diameter width Max & -- & -- & 2.94 & 0.95 & -- & -- \\
\hline RAA base diameter width Min & -- & -- & 1.91 & 0.85 & -- & -- \\
\hline
\end{tabular}

*: Significant

Table (4): Comparison between Right parasternal approach (3D) and Transesophageal approach (Gold standard)

\begin{tabular}{|l|c|c|c|c|c|c|}
\hline & \multicolumn{2}{|c|}{$\begin{array}{c}\text { Right parasternal } \\
\text { Approach(3D) }\end{array}$} & \multicolumn{2}{c|}{$\begin{array}{c}\text { Transeosophgeal approach } \\
\text { (Gold standard) }\end{array}$} & \multicolumn{2}{c|}{$\begin{array}{c}\text { Independent } \\
\text { t test }\end{array}$} \\
\cline { 2 - 7 } & Mean & SD & Mean & SD & t value \\
\hline SVC diameter Max(AP) & 1.90 & 0.57 & 1.73 & 0.54 & 1.246 & 0.217 \\
\hline SVC diameter Min(AP) & 1.41 & 0.51 & 1.46 & 0.61 & -0.320 & 0.750 \\
\hline SVC diameter Max (Lat) & 1.87 & 0.32 & 1.91 & 0.32 & -0.438 & 0.663 \\
\hline SVC diameter Min (Lat) & 1.56 & 0.52 & 1.39 & 0.44 & 1.180 & 0.244 \\
\hline CT width & 1.05 & 0.43 & 1.20 & 0.37 & 1.631 & 0.104 \\
\hline CT length & 0.64 & 0.23 & 0.62 & 0.23 & 0.315 & 0.754 \\
\hline RAA base diameter Max & 3.55 & 0.97 & 3.38 & 1.08 & 0.692 & 0.491 \\
\hline RAA base diameter Min & 2.27 & 0.96 & 2.57 & 0.69 & -1.440 & 0.155 \\
\hline RAA depth Max & 2.57 & 0.44 & 2.79 & 0.93 & 1.528 & 0.130 \\
\hline RAA depth Min & 2.01 & 0.62 & 2.16 & 0.87 & 1.055 & 0.318 \\
\hline RAA base diameter width Max & 2.25 & 0.95 & 1.98 & 0.48 & 1.192 & 0.236 \\
\hline RAA base diameter width Min & 1.91 & 0.85 & 1.55 & 0.64 & 1.680 & 0.100 \\
\hline
\end{tabular}




\section{Discussion}

The right atrium structure and function can be characterized by echocardiography, which provides diagnostic and prognostic information ${ }^{[10]}$. The proper usage of echocardiography could decrease the need for invasive techniques. Echocardiography is easily performed, relatively inexpensive and readily available ${ }^{[11]}$.

The current study was performed to assess feasibility and accuracy of two-and threedimensional transthoracic echocardiographic in patients with right atrial dilatation using the right parasternal approach in comparison with TEE as a gold slandered attending to cardiology department, AL-Azhar University Hospital (New Damietta) including 30 patients with right atrial dilatation.

The few previous trials reported that, right parasternal approach offers a different perception in evaluation of the right atrium structures and imaging of superior vena cava entrance into right atrium. Like standard 2DTTE, some subjects have superior view of right parasternal windows than others, and in these subjects, the structures are visualized and more detailed information obtained ${ }^{[12]}$.

Arisha et al. ${ }^{[2]}$ showed that imaging through right parasternal window was feasible in $52 \%$ of patients in their study, unlike our study where we were able to get the window in $60 \%$ of patients. This may be attributed to the inclusion criteria of our study group which required right atrial dilatation.

According to researchers' best of knowledge, no 2D or 3DTTE estimations were registered in normal subjects for superior vena cava, CT, or right atrium appendage in the available literature. Therefore, direct comparisons cannot be made. Autopsy values of RAA was done directly on human specimens and by computed tomography (CT) analysis, but these were done on non-beating soft hearts and thus, cannot be precisely compared with values obtained in vivo ${ }^{[2]}$.

Transthoracic echocardiography (TTE) is extensively used in evaluation of the cardiac function. The assessment are usually done according to different protocols with different probe positions which provide different views of the heart ${ }^{[13]}$.

In the present work, results confirmed the worth of 3DTTE over 2DTTE for evaluation of the right atrium structures. The imaging of SVC by 2DTTE in short axis is difficult, but 3DTTE permits the viewing of SVC in this axis even though the $3 \mathrm{D}$ dataset is obtained in long axis. In addition, transthoracic echocardiography 3DTTE was more accurate in assessment of the CT due to larger extent in a larger number of subjects could be imaged by 3DTTE. The 3DTTE values of SVC, CT, and RAA were larger than 2DTTE possibly reflecting better accuracy of 3D. All of these findings matched the study done by Arisha et al. ${ }^{[2]}$ in confirming that 3DTTE is superior to 2DTTE in showing right atrial structures

The advantage of TEE over TTE included clear images of structures that are difficult to view by transthoracic approach. Many structures could be assessed better with the TEE. However, TEE is a semi-invasive technique with well- known associated drawbacks and even when the RAA has been imaged by 2DTEE, most of the time its full extent is not viewed ${ }^{[15]}$.

In conclusion, results of the present work revealed the feasibility of parasternal approach in imaging right heart structures. In addition, 3DTTE had an incremental value over 2DTTE in the assessment of these structures.

\section{References}

1. Guerra VC, Coles J, Smallhorn JF. Aneurysm of right atrium diagnosed by 3-dimensional real-time echocardiogram. J Am Soc Echocardiogr. 2005; 18(11):1221. PMID: 16275533

2. Arisha MJ, Hsiung MC, Nanda NC, Gupta A, George DC, Elkaryoni A, Barssoum K, et al. Two- and three- dimensional transthoracic echocardiographic assessment of superior vena cava, crista terminalis, and right atrial appendage using the right parasternal approach. Echocardiography; 34 (12):1919-1929. PMID: 29226377

3. Marian AJ, Braunwald E. Hypertrophic cardiomyopathy: genetics, pathogenesis, clinical manifestations, diagnosis, and therapy.Circ Res. 2017; 121:749-770. PMID: 28912181

4. Denault AY, Langevin S, Lessard MR, Courval JF, Desjardins G. Transthoracic echocardiographic evaluation of the heart and great vessels. Can J Anaesth. 2018 Apr;65(4):449472. PMID: 29352414

5. Khoshhal S. Feasibility and effectiveness of threedimensional echocardio-graphy in 
diagnosing congenital heart diseases. Pediatr Cardiol. 2013 Oct; 34(7): 1525-31. PMID: 23677391

\section{Hadeed K, Hascoët S, Karsenty C, Ratsimandresy} M, Dulac Y, Chausseray G, et al. Usefulness of echocardiographicfluoroscopic fusion imaging in children with congenital heart disease. Arch Cardiovasc Dis. 2018 Jun - Jul;111(6-7):399-410. PMID: 29853351

7. Lancellotti P, Dulgheru R, Vannan M, Yoshida K. Heart valve disease (mitral valve disease): mitral regurgitation. The EACVI Textbook of Echocardiography, 2017; p.282.

8. Kawel-Boehm N, Maceira A, ValsangiacomoBuechel, Vogel-Claussen $\mathbf{J}^{4}$, Turkbey $\mathbf{E B}^{5}$, Williams R, et al. Normal values for cardiovascular magnetic resonance in adults and children. J Cardiovasc Magn Reson. 2015 Apr 18; 17:29. PMID: 25928314

9. Nagueh SF, Smiseth OA, Appleton CP, Byrd BF $3^{\text {rd }}$, Dokainish $H$, Edvardsen $T$, et al. Recommendations for the Evaluation of Left Ventricular Diastolic Function by Echocardiography: An Update from the American Society of Echocardiography and the European Association of Cardiovascular Imaging. Eur Heart J Cardiovasc Imaging. 2016; 17(12): 1321-1360. PMID: 27422899

10. Gupta DK. Assessment of the Atria. In: Essential Echocardiography, Scott D. Solomon, Justina C. Wu and Linda D. Gillam (eds); Elsevier Inc. 2019; pp 190-194

11. Markley RR, Ali A, Potfay J, Paulsen W, Jovin IS. Echocardiographic Evaluation of the Right Heart. J Cardiovasc Ultrasound. 2016;24 (3): 183-190. PMID: 27721944

12. Patel V, Nanda NC, Upendram S, Enar S, Mehmood F, Vengala $S$, Frans E, Bodiwala K. Live three-dimensional right parasternal and supraclavicular transthoracic echocardio-graphic examination. Echocardiography. 2005; 22:349-360. PMID: 15839993

Lang RM, Badano LP, Mor Avi V, Afilalo J, Armstrong A, Ernande L, et al. Recommendations for cardiac chamber quantification by echocardiography in adults: An update from the Americn Society of Echocardiography and the European Association of Cardiovascular imaging. Eur Heart J Cardiovasc Imaging 2015; 16:233271. PMID: 25712077.
14. Sherifi I, Omar AM, Varghese M, Weiner M, Anyanwu A, Kovacic JC, et al. Comparison of transesophageal and transthoracic echocardiography under moderate sedation for guiding transcatheter aortic valve replacement Echo Res Pract. 2018; 5(2): 7987. PMCID: PMC5987181

15. Subramaniam B, Riley MF, Panzica PJ, Manning WJ. Transesophageal echocardiographic assessment of right atrial appendage anatomy and function: comparison with the left atrial appendage and implications for local thrombus formation. J Am Soc Echocardiogr. 2006; 19:429-433. PMID: 16581482. 